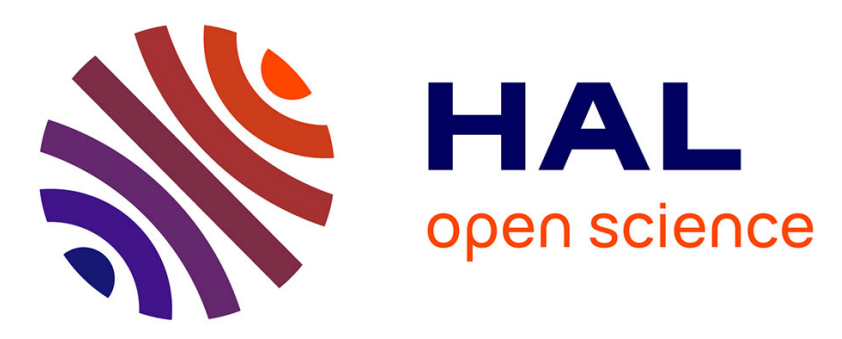

\title{
Stabilization of Supramolecular Polymer Phase at High Pressures
}

\author{
Nikolaos Burger, Antonios Mavromanolakis, Gerhard Meier, Patrick \\ Brocorens, Roberto Lazzaroni, Laurent Bouteiller, Benoit Loppinet, Dimitris \\ Vlassopoulos
}

\section{To cite this version:}

Nikolaos Burger, Antonios Mavromanolakis, Gerhard Meier, Patrick Brocorens, Roberto Lazzaroni, et al.. Stabilization of Supramolecular Polymer Phase at High Pressures. ACS Macro Letters, 2021, pp.321-326. 10.1021/acsmacrolett.0c00834 . hal-03142934

\section{HAL Id: hal-03142934 \\ https://hal.science/hal-03142934}

Submitted on 16 Feb 2021

HAL is a multi-disciplinary open access archive for the deposit and dissemination of scientific research documents, whether they are published or not. The documents may come from teaching and research institutions in France or abroad, or from public or private research centers.
L'archive ouverte pluridisciplinaire HAL, est destinée au dépôt et à la diffusion de documents scientifiques de niveau recherche, publiés ou non, émanant des établissements d'enseignement et de recherche français ou étrangers, des laboratoires publics ou privés. 


\title{
Stabilization of supramolecular polymer phase at
}

\section{high pressures}

\author{
N. A. Burger ${ }^{1,2}$, A. Mavromanolakis ${ }^{1}$, G. Meier $^{3}$, \\ P. Brocorens ${ }^{4}$, R. Lazzaroni ${ }^{4}$, L. Bouteiller ${ }^{5}$, B. Loppinet ${ }^{1}$, D. Vlassopoulos ${ }^{1,2}$
}

1 Foundation for Research \& Technology Hellas (FORTH), Institute for Electronic Structure \& Laser, Heraklion 70013, Greece

2 University of Crete, Department of Materials Science \& Technology, Heraklion 70013, Greece 3 Forschungszentrum Jülich, Institute of Complex Systems (ICS-3), 52425 Jülich, Germany 4 University of Mons, Laboratory for Chemistry of Novel Materials, Materials Research Institute, 7000 Mons, Belgium

5 Sorbonne Université, CNRS, IPCM, Equipe Chimie des Polymères, 75005 Paris, France

\begin{abstract}
We utilize dynamic light scattering (DLS) and passive microrheology to examine the phase behavior of a supramolecular polymer at very high pressures. The monomer, 2,4-bis(2ethylhexylureido)toluene (EHUT), self-assembles into supramolecular polymeric structures in the non-polar solvent cyclohexane, by means of hydrogen bonding. By varying concentration and temperature at atmospheric pressure, the formation of viscoelastic network (at lower temperatures) and predominantly viscous phases, based on self-assembled tube and filament structures respectively, has been established. The associated changes in the rheological properties have been attributed to a structural thickness transition. Here, we investigate the effects of pressure variation, from atmospheric up to $1 \mathrm{kbar}$. We construct a temperature-pressure diagram that reveals the predominance of the viscoelastic network phase at high pressures. The transition from viscoelastic network organization of the tubes to a weaker viscous-dominated
\end{abstract}


structure of the filaments is rationalized by using the Clapeyron equation which yields an associated volume change of about $8 \AA^{3} /$ molecule. This observation is rationalized by means of Molecular Dynamics simulations of the two phases, which show a decrease in the molecular volume at the filament-tube transition, originating from increased intermolecular contacts in the tube with respect to the filament. These new findings offer insights into the role of pressure in stabilizing self-assemblies.

\section{INTRODUCTION}

Pressure is often considered to be a forgotten thermodynamic variable, in part because pressuredependent experimentation is far more challenging than its temperature-dependent counterpart. Yet, over the years several investigations of static and dynamic properties of soft materials at high pressures have been reported in the literature. For example, moderate pressures of the order of 100 bar were found to influence the second virial coefficient and radius of gyration of flexible polymers in different solvents. ${ }^{1}$ Increasing pressure (up to about 4 kbar) was reported to increase the intrinsic viscosity of polymer solutions, with the Huggins coefficient exhibiting a nonmonotonic dependence with a broad minimum. ${ }^{2}$ Clearly, pressure affects the miscibility in polymer mixtures, promoting miscibility in LCST polymer solutions ${ }^{3}$ and in UCST polymer blends ${ }^{4}$ due to volume changes on mixing. More complicated is the effect of pressure on triblock copolymer micelles, where it is found to promote crystal to liquid transition, but often the micellar liquid is topologically interacting. ${ }^{5}$ Furthermore, pressure is found to affect the glass transition and associated alpha relaxation (typically increasing the glass temperature) in supercooled liquids ${ }^{6}$ and a wide range of shape-persistent polymeric materials with intrinsic orientational order, for which the lack of thermal energy rather than free volume is proposed to be responsible for vitrification [7]. In general, soft materials experience high pressures in a variety of applications or during their transformation, which renders relevant studies necessary. For example, the application of pressure was found to increase the yield stress and strain of polymeric solids undergoing tensile deformation. ${ }^{8}$ Additional examples involve processing and capillary flow of polymer melts, ${ }^{9-14}$ drilling operations with fracturing fluids, ${ }^{15}$ the use of high 
pressure to obtain protein crystals from spider silk ${ }^{16}$ and the high-pressure preparation of dairy products (emulsions). ${ }^{17}$

Despite the above advances, it appears that the role of pressure on the properties of selfassemblies, and in particular their phase behavior, has not received much attention. Recently, pressure was reported to stabilize dynamic supramolecular assemblies of the host-guest type (enhancing their binding equilibria) and maintaining a constant viscosity (in contrast to crosslinked fluids, which may suffer pressure-induced viscosity thinning). ${ }^{18}$ That work shows that pressure is an important variable that may affect supramolecular associations and exploiting its consequences is an outstanding challenge. In this letter, we present a methodology to address this challenge by using as archetype a reversible supramolecular polymer built from 2,4-bis(2ethylhexylureido)toluene (EHUT), based on a bis-urea moiety. Its synthesis, thermodynamic and rheological properties have been discussed quite extensively in the literature. ${ }^{19-29}$ In non-polar solvents such as toluene, cyclohexane or dodecane, at high enough concentrations EHUT selfassembles into long supramolecular polymers, which have tube structure at lower temperatures and filament structure at higher temperatures. Their viscoelastic behavior is similar to wormlike surfactant micelles whose rheology depends on the bonding lifetime and overall length (which controls terminal relaxation). ${ }^{20,23-30}$ A similar bis-urea was recently found to exhibit thickening of high-pressure (about 345 bar) supercritical propane with significant implications in fracturing fluid processing for enhanced oil recovery operations. ${ }^{31}$

Here we investigate the effects of pressure on the thermoreversible supramolecular assemblies of EHUT in cyclohexane, which combine different length and time scales depending on temperature and concentration.

\section{MATERIALS}

The synthesis of EHUT was achieved by reacting racemic 2-ethylhexylamine with 2,4-toluene diisocyanate [20]. An apolar solvent, cyclohexane (99.7\% pure) was used as received, obtained from Sigma Aldrich. Its dielectric constant is 2.02.32 The solutions were prepared under conditions of atmospheric humidity and room temperature, by adding the EHUT powder to the solvent and stirring for at least 48 hours. ${ }^{27}$ 


\section{METHODS}

\section{III.A. High-Pressure DLS}

A homemade high-pressure cell was coupled to a single scattering DLS setup (from ALV, Germany) for the high-pressure DLS (HP-DLS) experiments (Fig.1). Here we provide a brief description of the main components: the pressurized chamber comprises 6 optical windows corresponding to scattering angles of 45,90 and $130^{\circ}$, and the light beam was focused with an 50x objective lens before reaching the sample, in the middle of the cylindrical cell. Nitrogen was compressed by means of a membrane compressor and entered the cell from the top. Details concerning the cell, windows and sealing are described elsewhere. ${ }^{33,34}$ The experiments were performed at pressures ranging from 1 to 1200 bar and temperatures from 20 to $70^{\circ} \mathrm{C}$ (the latter were controlled by means of a recirculating water/alcohol bath and measured with a thermocouple attached at the surface of the sample cell). Since the pressure-transmitting medium is compressed nitrogen, special care was taken for the measurement time and the selected sample volume in order to avoid diffusion of nitrogen molecules into the polymer sample up to the measurement point (where the laser beam enters the sample). More specifically, the diffusion coefficient of nitrogen molecules in liquids such as organic solvents, at ambient conditions $(\mathrm{P}=$ 1 bar, $\mathrm{T}=20^{0} \mathrm{C}$ ) is in the order of $10^{-9} \mathrm{~m}^{2} / \mathrm{s},{ }^{35}$ therefore for distances of about $1 \mathrm{~cm}$ (between the

free surface of the solution and the measurement point), the corresponding time $t=\frac{\left\langle r^{2}\right\rangle}{2 D}$, is in the order of days, ensuring that the measurements are unaffected by possible dilution of the sample. Further evidence is provided in Fig.S1 of the supporting information (SI) which shows identical where DLS data over long times for a similar system (EHUT in dodecane). A cw laser at $514.5 \mathrm{~nm}$ was used and the scattering angle was fixed at $\theta=90^{\circ}$. A mono-mode optical fiber was employed to feed the scattered light into an avalanche photodiode (Perkin-Elmer) operating in photon counting mode (Fig. 1). The time autocorrelation function of the scattering intensity was obtained in real time by means of an ALV 5000E digital correlator, and the electric field autocorrelation function (or intermediate scattering function, ISF) $C(t)$ was recorded and analyzed (see also the supporting information, SI and Fig.S2). The pressure was kept constant over long times and data were highly reproducible. 
To better appreciate the difference in dynamics between tube (viscoelastic network) and filament (predominantly viscous) phases, high-pressure microrheology experiments ${ }^{36,37}$ were conducted as well. To this end, polymethylmethacrylate (PMMA) particles were added to an EHUT solution in cyclohexane at a volume fraction of about $10^{-4} \mathrm{vol} \%$, to act as effective tracers for following their motion. They were chemically grafted with polyhydroxy stearic acid (PHSA) chains (of about $10 \mathrm{~nm}$ in length) to ensure stability of the dispersion, their hydrodynamic radius was $\mathrm{R}=130 \mathrm{~nm}$ (measured in the dilute regime by dynamic light scattering, DLS) and their polydispersity was $10 \%{ }^{38}$ The dual goal of adding these particles was to stay in the single scattering limit (i.e., photons reaching the instrument detector being scattered by a probed particle and not also re-scattered by neighboring particles ${ }^{39}$ and reach a scattering intensity at least 50 times higher compared to that of EHUT solutions in the absence of particles. Details of the microrheological measurements are presented in the SI (see also Fig.S3). Note that our approach is similar to that of Ref.36 but differs in the pressurizing system (we used gas instead of oil) and the detection method (we used DLS in the single scattering instead of diffusive wave limit). 


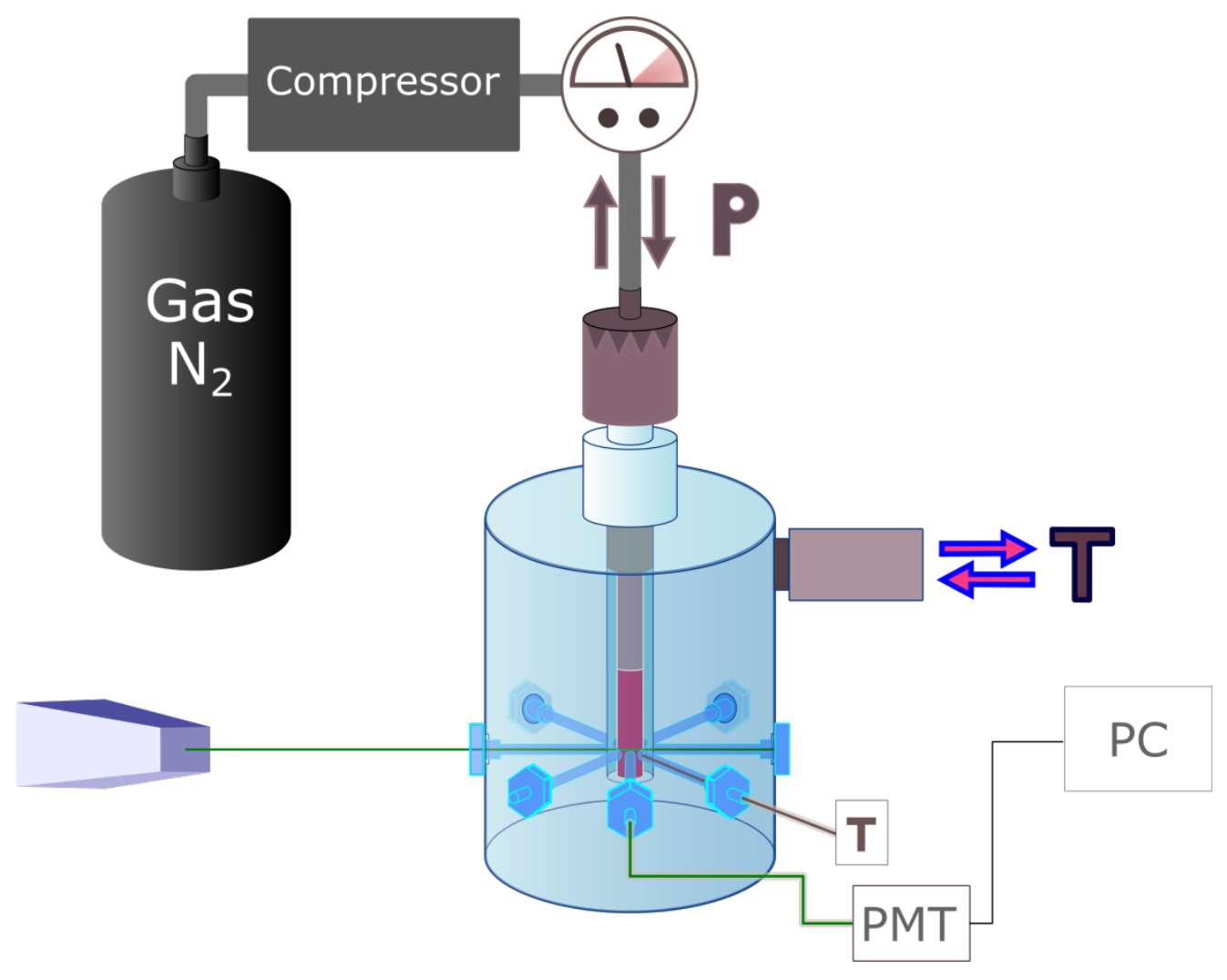

Figure 1: Schematic illustration of the experimental high-pressure DLS setup. Shown, out of scale, are: the gas (nitrogen) tank, compressor, chamber cell with different glass windows for scattering at different fixed angles, gas inlet to DLS cell (P), inlet of recirculating fluid to cell chamber for maintaining constant temperature ( $\mathrm{T}$ with arrows), temperature meter $(\mathrm{T})$, laser (with green incident beam), scattered beam entering the photomultiplier tube (PMT), whose signal is collected and analyzed by a correlator on a PC.

\section{III.B. Molecular Dynamics simulations}

Molecular Dynamics (MD) simulations were performed with the Materials Studio (MS) software on a tube and a helical filament of EHUT molecules. ${ }^{23,40,41}$ Details are given in the SI. The volumes of conformations generated during the simulations were determined by rolling on the molecules a dummy atom that acts as a spherical probe (Fig. S7). The contact is defined between the Van der Waals radius of the probe and the Van der Waals radii of the atoms (as implemented in the Dreiding force field ${ }^{42}$ used to do the MD simulations). The radius of the probe can be chosen arbitrarily: (i) if it is set to zero, the probe simply explores the surface defined by the Van der Waals radii of the atoms in the molecule and the corresponding volume is called the Van der 
Waals volume (see gray area in Fig. S7); (ii) if the radius of the probe is non-zero, some parts of the molecular surface become inaccessible to the probe, its trajectory then defines a Connolly surface (see the black curve in Fig. S7) and the corresponding volume is called the Connolly volume. Its value depends on the radius of the probe. In this work, the radius of the probe was given different values: $0 \AA, 0.8 \AA, 1.6 \AA$ (typical of a hydrogen atom), and $2.4 \AA$. In addition to the tube and helical single filament, a molecule was extracted from the simulated assemblies, and its conformation was used to evaluate its van der Waals and Connolly volumes in the absence of packing with neighbors, i.e., as an isolated molecule. For the three systems, the volumes were calculated on six different conformations and averaged.

\section{RESULTS AND DISCUSSION}

The phase diagram of EHUT solutions in cyclohexane is schematically depicted in Fig.2. Depending on the concentration and temperature, EHUT solutions may be monomeric (unassembled) or form self-assembled filaments or tubes, under atmospheric pressure, qualitatively similar to the respective behavior in toluene. ${ }^{22,23,43}$ Both filament and tube structures are long chains of molecules held together by hydrogen bonds, but the cross-section of the former contains a single EHUT monomer, whereas that of the latter contains three monomers, which leads to a slowing-down of the chain scission process and eventually to viscoelastic behavior. Of interest in this work is the specific concentration $\mathrm{c}=4 \mathrm{~g} / \mathrm{L}$, where a tube-to-filament transition is observed at $49^{\circ} \mathrm{C}$. In fact, SANS and IR spectroscopy experiments indicate a structural thickness transition at $\mathrm{T} \approx 52^{\circ} \mathrm{C}, \mathrm{P}=1$ bar and $\mathrm{c}=10.4 \mathrm{~g} / \mathrm{L}$. ${ }^{44}$ Characterization details of the tubefilament transition at low pressures are given in Table S1 of the SI. The effects of pressure will be discussed below. Note that there is no macroscopic signature of phase separation in this solvent, as the samples remain visually transparent. We have also measured the pressuredependent solvent viscosity (see Fig.S4 of the SI) and accounted for it, as well as for the respective temperature dependence in the presentation of the experimental data in order to emphasize the net effect of pressure on the supramolecular polymer organization. Indeed, in Fig.3 below the dynamic light scattering data or dynamic viscoelastic data have been appropriately normalized by the solvent viscosity (in their time or frequency axes, respectively) at the respective pressure and temperature (see also in Fig.S5 of the SI the effect of pressure on 
the relative viscosity of a $4 \mathrm{~g} / \mathrm{L}$ EHUT/cyclohexane solution). Moreover, since the thrust of the present investigation is the effects of pressure on the EHUT-based reversible supramolecular polymers, we work in conditions of ambient humidity without considering its effects on the viscoelastic properties of the solutions. ${ }^{27}$



Figure 2: Schematic illustration of the phase diagram for EHUT solutions in cyclohexane at atmospheric pressure. Three regimes are identified: monomeric (unassembled), self-assembled thin filaments and self-assembled tubes. The cartoons illustrate the molecular structure of the EHUT monomer, and the self-assembled filaments and tubes (the dotted lines illustrate the links (hydrogen bonds) between units which are shown by circles). The red filled circle indicates the observed structural transition from filament to tube for $\mathrm{c}=4 \mathrm{~g} / \mathrm{L}$ and $\mathrm{T}=49{ }^{0} \mathrm{C}$, which is in excellent agreement with the literature ${ }^{44}$ (see also Table 1).

First, we investigated the dynamics at atmospheric pressure. As can be seen in Fig.3a, there is a transition from unimodal ISF at high temperatures $\left(\mathrm{T} \geq 49^{\circ} \mathrm{C}\right)$ to bimodal ISF at low temperatures $\left(\mathrm{T}<49^{\circ} \mathrm{C}\right)$. A quantitative analysis of the relaxation modes (amplitutes and times) is presented the SI (Figs. S2,S6 and Table S2). We attribute the presence of a second, slow relaxation process to the appearance of self-assembled EHUT tubes, which are known to exhibit strong viscoelasticity accompanied by a plateau modulus ${ }^{26-28}$; note that entangled polymers have been reported to reveal a second slow mode in their ISF, assigned to the relaxation of the entanglement 
network. ${ }^{45-47}$ Note further that the second mode is readily observed by eye. Hence, single-mode ISF refers to filaments and bimodal ISF to tubes. Moreover, this distinction is also corroborated by the total scattering intensity as a function of temperature, which is depicted in the inset of Fig.3a upon heating and cooling at 1 bar; the tube-to-filament transition is marked by a change in intensity. Note that in some cases (higher pressure or lower temperature) a weaker third slower mode is detected (Table S2), which reinforces the assignment to tube phase. Consequently, we use the existence of slow mode(s) in the ISF as a signature of the formation of tubes, in order to construct a phase diagram, which is depicted in Fig.4 below. To this end we examine the shape of the ISF of a given EHUT/cyclohexane solution $(c=4 \mathrm{~g} / \mathrm{L})$ at different temperatures and pressures (see also Fig.S6 and Table S2 of the SI). For example, the typical ISFs at three temperatures and $\mathrm{P}=325$ bar (Fig. $3 \mathrm{~b}$ ) indicate that at $49^{\circ} \mathrm{C}$ we have a tube phase while at $58^{0} \mathrm{C}$ (where the slow mode progressively disappears) we have a filament phase, hence there is a shift of the tube-filament transition from about $49^{\circ} \mathrm{C}$ (at atmospheric pressure, Fig. 3 a) to about $58^{\circ} \mathrm{C}$ (at 325 bar), where the slow mode progressively disappears. At 600 bar, the supramolecular solution forms only tubes throughout the examined temperature range (Fig.3c). Furthermore, an examination of the microrheological linear viscoelastic spectrum indicates sharp differences between low (1 bar) and high (600 bar) pressures, i.e., filaments and tubes (Fig.3d and Fig.S5 of the SI). At this particular semidilute concentration of $4 \mathrm{~g} / \mathrm{L}$, a loose arrangement of selfassembled filaments is formed at $49^{\circ} \mathrm{C}$ and 1 bar (see also Fig.2 and Ref.27), which exhibits the rheological signature of a predominantly viscous liquid (with low viscosity of $\sim 3 \mathrm{mPas}$, see Fig.S5 of the SI) The same response is expected at higher temperatures, well into the phase dominated by filaments. However, on increasing the pressure to $600 \mathrm{bar}$ at the same temperature, the viscoelastic spectrum changes drastically, signifying the response of an entangled network of supramolecular $\operatorname{tubes}^{27}$ (and a slow mode in the ISF). The eventual terminal regime of this network gives rise to a viscosity which exceeds that of the filaments by a factor of about 330 (Fig.S5). Finally, we note that for both tubes and filaments, the EHUT solutions were completely transparent without visual hint of phase separation. The interesting finding of Fig. 4 is that high pressures appear to stabilize further the tubes, which are the only self-assembled structure at this concentration. It is also important to emphasize that the observed tube-filament transitions are reversible and all measurements are performed in equilibrium 
conditions (very fast kinetics due to the dynamic character of this supramolecular system). Finally, we note that the high-frequency viscoelastic response of tubes and filaments in Fig.3d compares favorably with that of wormlike surfactant micelles and other semiflexible polymeric materials. The latter were reported to exhibit an intermediate and high-frequency power-law dependence of G" with exponents of 5/9 (Rouse-Zimm) and 3/4 (internal bending modes), respectively, with the transition occurring at the scale of the persistence length, as well as good agreement between conventional rheometry and passive microrheology data. ${ }^{48,49}$ The analysis of the high-frequency response is presented in the SI (Fig.S7).
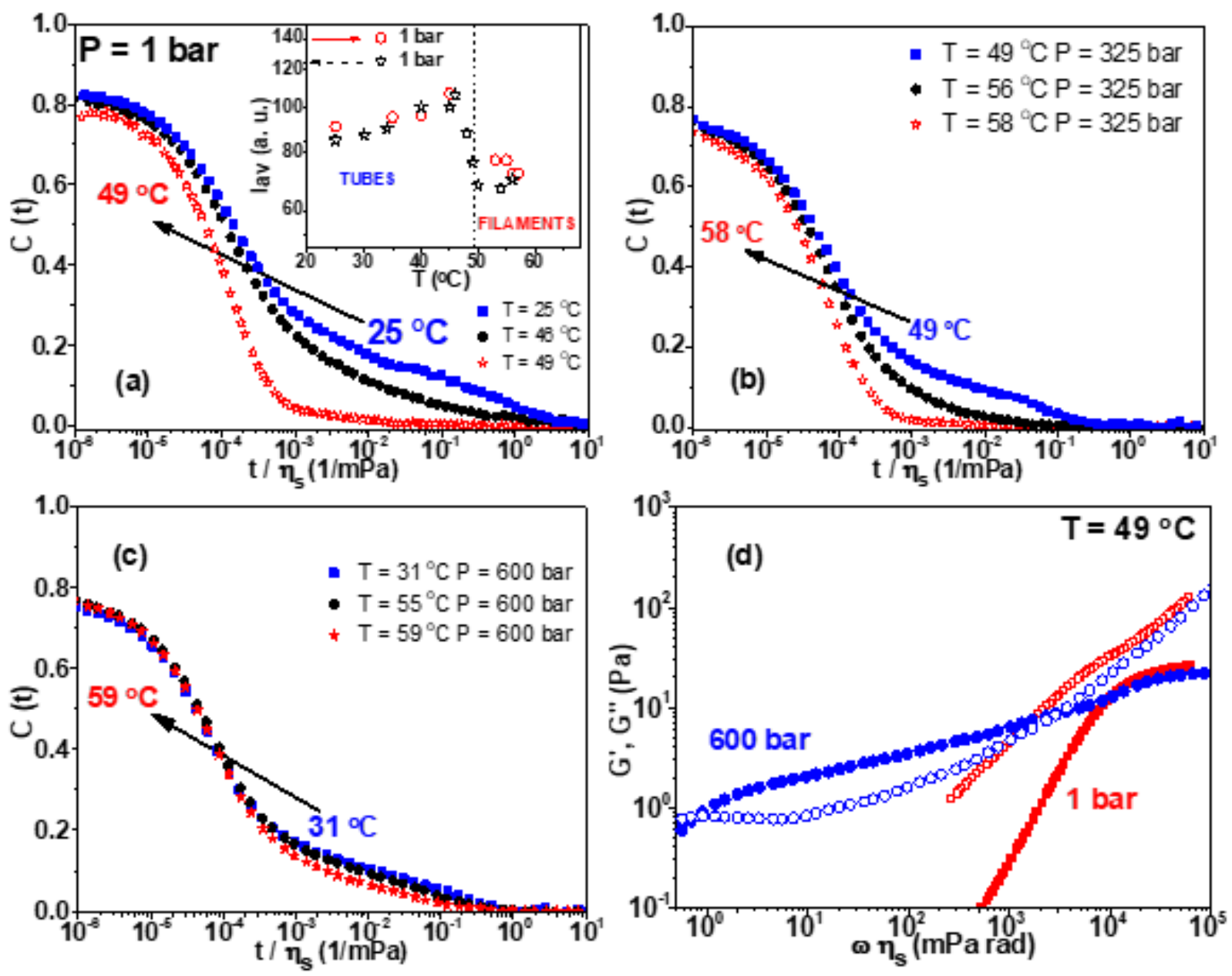

Figure 3: Experimental data of EHUT/cyclohexane solution at $4 \mathrm{~g} / \mathrm{L}$, with the time divided by the temperature- or pressure-dependent solvent viscosity (see also SI). (a) Intermediate scattering functions (ISF); the open (filled) symbols correspond to filament (tube) structures. Heating from 
$25^{\circ} \mathrm{C}$ (two modes) to $49{ }^{\circ} \mathrm{C}$ (one mode) at 1 bar in the direction of the arrow. Inset: Average total scattering intensity at different temperatures and pressure of $1 \mathrm{bar}$; the red data and arrow indicate the direction of heating, whereas the black data and dashed arrow the cooling direction. (b) ISF for different temperatures upon heating from $49^{\circ} \mathrm{C}$ to $58^{\circ} \mathrm{C}$ at 325 bar (direction of arrow). The former corresponds to tubes and the latter to filaments. The intermediate temperature of $56^{\circ} \mathrm{C}$ is transitional, likely with a mixture of tubes and filaments but predominance of the former because of the presence of the slow mode. (c) Respective ISF data on heating from $31^{\circ} \mathrm{C}$ to $59^{\circ} \mathrm{C}$ at 600 bar (direction of arrow). (d) microrheological viscoelastic spectra at $49^{\circ} \mathrm{C}$ and two different pressures, 1 bar and 600 bar, corresponding to the limit of filaments (red squares) and to tubes (blue circles), respectively. Open (filled) symbols refer to loss modulus G" (storage modules G'). The closeness of the high-frequency data suggests that local processes do not have substantial, if any, dependence on pressure (see also SI and Fig.S7). The tube data exhibit typical response of a viscoelastic network and the filaments data reflect the behavior of a viscous liquid. The respective data without the scaling of the time are shown in Fig.S6 of the SI.

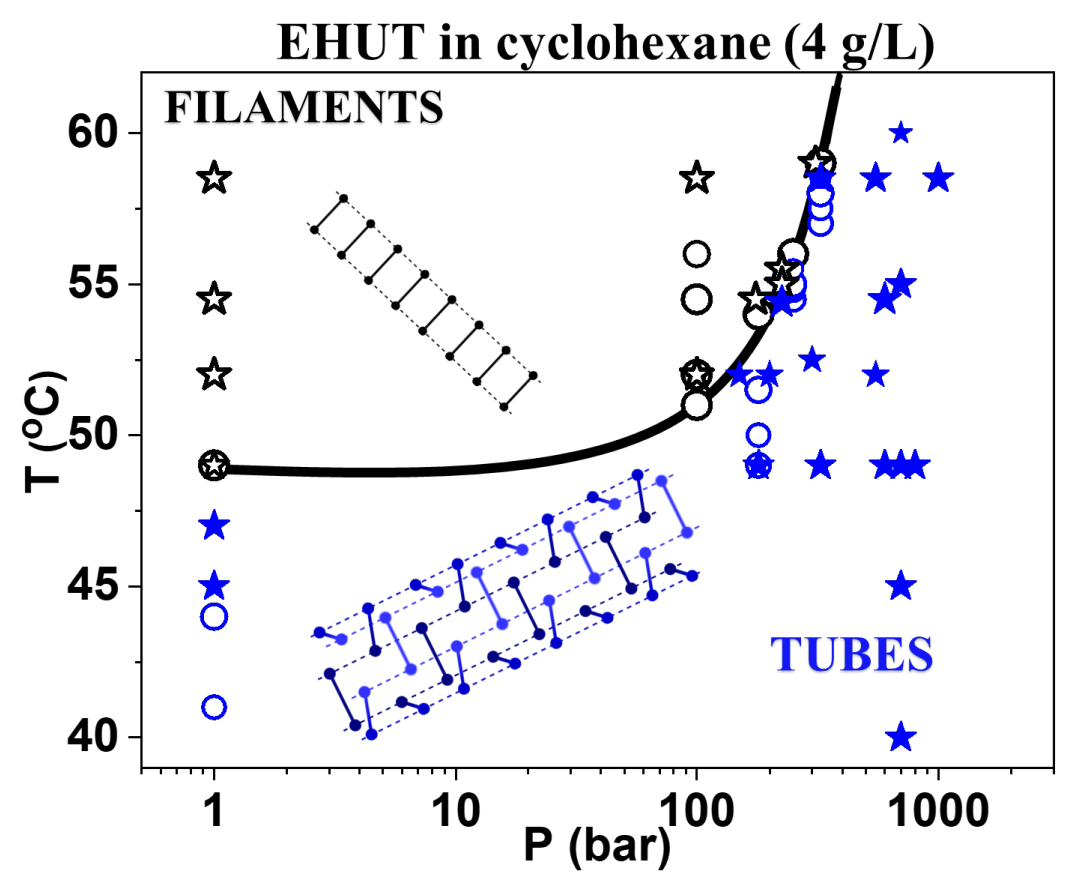

Figure 4. Phase diagram of a $4 \mathrm{~g} / \mathrm{L}$ EHUT / cyclohexane solution in the (T, P) space. Star symbols correspond to passive microrheological data (see Fig.S2 in the SI). Circles correspond to DLS (without added particles). The black line is drawn to guide the eye. Blue (black) color indicates tubes (filaments).

In general, the effect of pressure can be accounted for by approximating it as a first order transition and invoking the Clapeyron equation

$$
\frac{d P}{d T}=\frac{\Delta H}{T^{*} \Delta V}
$$


where $T^{*}$ is the tube-filament transition temperature at reference (atmospheric pressure), $\Delta H$ is the latent heat per molecule associated with the tube-to-filament transition and $\Delta V$ the associated difference in molecular volumes. The experimental results of Fig.4 suggest that upon increasing the pressure to 300 bars, i.e., $\Delta P \approx 300$ bar, the transition temperature approaches $60^{\circ} \mathrm{C}$, i.e., $\Delta T \approx 10 K$. This suggests that $\frac{\Delta H}{\Delta V} \approx 9.7 \times 10^{8} \mathrm{~Pa}$. Note that $\Delta H$ is about $1.7 k_{B} T$ at low pressures (see Table S1 and Ref.47), and we assume to a first approximation that it has the same dependence on pressure with $\Delta V$. This leads to $\Delta V \approx 8 \AA^{3} /$ molecule.

In order to rationalize that volume change, MD simulations were carried out on model systems for the filament and the tube (see details in SI), along with calculations on an isolated EHUT molecule as reference. The simulations indicate that the van der Waals volume decreases from the isolated molecule to the filament, as the interactions between the molecules, especially the formation of $\mathrm{H}$ bonds, leads to an overlap of the van der Waals volumes (see also Fig.S7). The volume is further reduced when the filament evolves to a tube, on the order of $10 \AA^{3} /$ molecule, which conforms to the experimental results (Table 1). The volume decrease from filament to tube reflects the increase of contacts between neighbors. In both structures, the hydrogen bonds between urea moieties drive the assembly, but in the filament, the cores interact by $\pi-\pi$ stacking and segregate from the alkyl chains that protrude in the environment, while in the tube, the cores and alkyl chains intercalate to form a continuous, dense medium minimizing its exposure to the environment (Fig. 5). .

Table 1: Average van der Waals volume per molecule with standard deviation, for the tube, the filament and the isolated molecule.

\begin{tabular}{|l|c|c|c|c|}
\hline & $\begin{array}{c}\text { van der Waals volume per } \\
\text { molecule }\left(\AA^{3}\right)\end{array}$ & \multicolumn{2}{|c|}{ Connolly volume per molecule $\left(\AA^{3}\right)$} \\
\hline & $638.3 \pm 0.7$ & $0.8 \AA$ probe & $1.6 \AA$ probe & $2.4 \AA$ probe \\
\hline Tube & $649.3 \pm 0.8$ & $689.8 \pm 1.6$ & $729.9 \pm 2.6$ & $766.3 \pm 2.9$ \\
\hline Filament & $670.4 \pm 2.8$ & $701.6 \pm 1.4$ & $763.9 \pm 2.7$ & $805.6 \pm 3.5$ \\
\hline Isolated molecule & $688.1 \pm 3.6$ & $714.8 \pm 4.7$ & $741.0 \pm 6.0$ \\
\hline
\end{tabular}



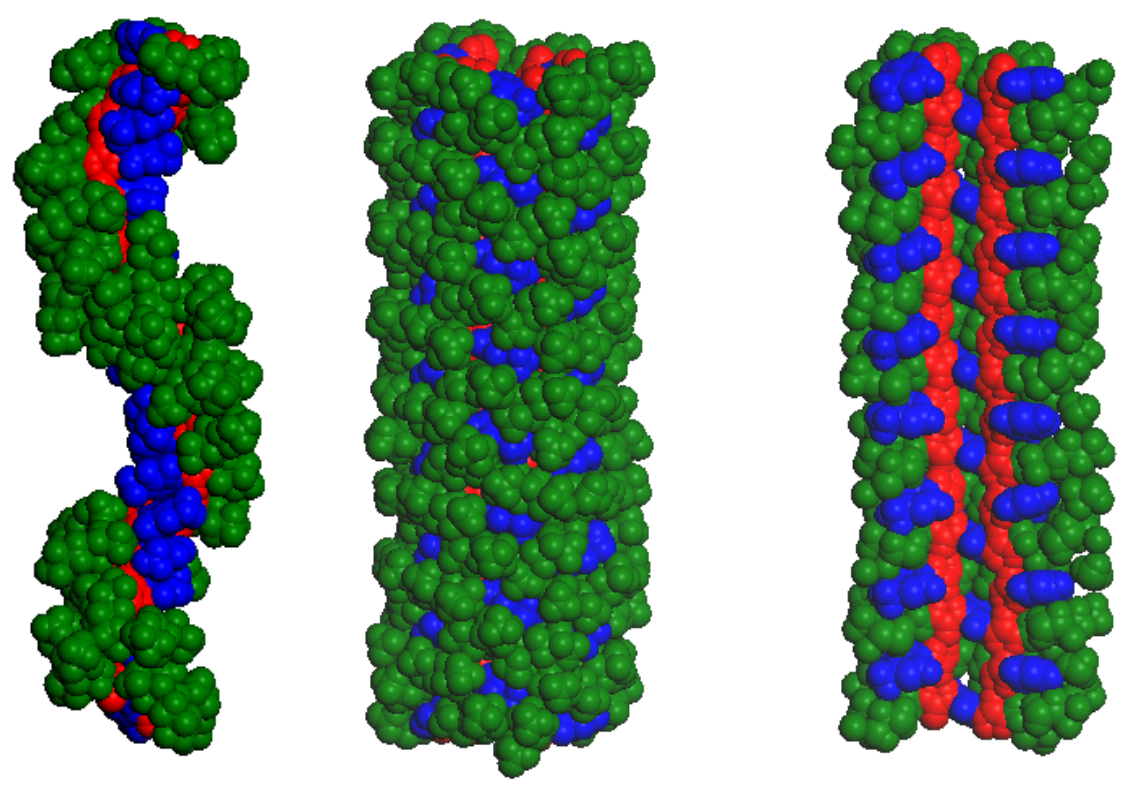

Figure 5. Pieces of filament (left), tube (center), and tube longitudinally cut in two to expose its inner part (right). The assemblies are of the same length ( $72 \AA)$; the atoms are rendered as spheres having the Van der Waals radius of the elements (CPK representation). The urea moieties are in red, the toluene cores in blue, and the alkyl chains in green.

In this approach, the possible reorganization of the solvent has not been considered explicitly. Nevertheless, we investigated the accessibility of cavities in the EHUT assemblies to solvent molecules, we used probes with different radii and found that an isolated molecule has the smallest Connolly volume, while it has the largest van der Waals volume, when compared to the two assemblies. The larger Connolly volume in the assemblies is explained by the formation of new cavities inaccessible to solvent upon molecular packing. When comparing the Connolly volumes of the filament and tube, the volume changes vary with the probe size, as expected. More importantly, the tubes always appear more compact (i.e., have a higher density) than the filaments (see Table 1 and Fig. S8), again pointing to the higher density of intermolecular contacts in the tubes.

An important question is whether the stabilization of the tube structure at high pressures reflects a kind of host-guest effect, due to more favorable interactions between the tube and the included 
solvent, or if it is a more general phenomenon. Concerning the former situation, we note that solvent interactions inside and outside the tubes have been found to be different. ${ }^{49}$ In the latter case, it should also occur in other hydrogen-bonded assemblies by enhancing bonding at high pressures. Another challenge is to determine the consequences of the above pressure-dependent structural changes on the viscoelastic properties of the supramolecular assemblies. These questions will be addressed in the future.

In summary, high-pressure DLS in the single scattering limit and passive microrheology were employed to construct the PT phase diagram of a supramolecular polymer. The 2,4-bis(2ethylhexylureido)toluene (EHUT) self-assembles into stable viscoelastic network tube or viscous filament phases in apolar solvent cyclohexane, depending on temperature and concentration. By choosing a specific concentration, we spanned the temperature-pressure parameter space and identified tubes and filaments. We found that pressure substantially affects the phase behavior by promoting the tube phase. Indeed, at high levels of pressure (in the range $600 \mathrm{bar}$ ) the tube phase is stabilized throughout the entire range of temperatures. The tube-tofilament transition is rationalized as a quasi-first order transition and the associated volume change, in the order of $10 \AA^{3} /$ molecule, can be ascribed to a higher density of intermolecular contacts in the tube, as determined is consistent with independent findings from molecular modeling simulations.

\section{ACKNOWLEDGMENTS}

Partial support has been received by the European Commission (Horizon2020-INFRAIA-20161, EUSMI grant no. 731019) and the Greek Secretariat for Research and Technology (INNOVATION program-AENAO). We thank J. K. G. Dhont (Forschungszentrum Jülich) for donation of part of the instrumentation and A. Vagias (Technical University of Munich) for early experimental tests. The modelling activities are supported by FNRS EOS 2Dto3D project (grant 30489208) and Consortium des Équipements de Calcul Intensif - CÉCI (Grant 2.5020.11) and by the Walloon Region (ZENOBE Tier-1 supercomputer, grant 1117545). 


\section{REFRENCES}

[1] Gaeckle, D. ; Patterson, D. Effect of Pressure on the Second Virial Coefficient and Chain Dimensions in Polymer Solutions. Macromolecules 1972, 5, 136-141.

[2] Schmidt, J. R. ; Wolf, B. A. Pressure Dependence of Intrinsic Viscosities and Huggins Constants for Polystyrene in tert-Butyl Acetate. Macromolecules 1982, 15, 1192-1195)

[3] Wolf, B. A. Improvement of polymer solubility: Influence of shear and of pressure, Pure \& Appl. Chem. 1997, 69, 929-933.

[4] Beiner, M.; Fytas, G. ; Meier, G.; Kumar, S. K. Pressure-Induced Compatibility in a Model Polymer Blend, Phys. Rev. Lett. 1998, 81, 594-597.

[5] Kloxin, C. J. ; van Zanten, J. H. High-pressure phase diagram of an aqueous PEO-PPO-PEO triblock copolymer system via probe diffusion measurements, Macromolecules 2010, 43(4), 2084-2087.

[6] Patkowski, A.; Paluch, M.; Kriegs, H. Dynamic light scattering studies of supercooled phenylphthalein-dimethylether dynamics under high pressure, J. Chem. Phys., 2002, 117(5), 2192-2198.

[7] Floudas, G. Effects of pressure on systems with intrinsic orientational order, Prog. Polym. Sci. 2004, 29, 1143-1171.

[8] Christiansen, A. W.; Baer, E.; Radcliffe, S. V. The mechanical behavior of polymers under high pressure, Philosophical Magazine, 1971, 24:188, 451-467.

[9] Guan, Q.; Shen, K.; Li, L.; Zhu, J. Structure and properties of self-reinforced polyethylene prepared by oscillating packing injection molding under low pressure, J. App. Polym. Sci. 1995, 55, 1797-1804.

[10] Berthe, D.; Vergne, P. High pressure rheology for high pressure lubrication: A review, $J$. Rheol. 1990, 34, 1387-1414.

[11] Couch, M. A. ; Binding, D. M. High pressure capillary rheometry of polymeric fluids. Polymer 2000, 41(16), 6323-6334.

[12] Hatzikiriakos, S. G.; Dealy, J. M. Start-up Pressure Transients in a Capillary Rheometer, Polym. Eng. Sci. 1994, 34(6), 493-499. 
[13] Mackley, M. R. ; Spitteler, P. H. J. Experimental Observations on the Pressure-Dependent Polymer Melt Rheology of a Linear Low-Density Polyethylene using a Multipass Rheometer, Rheol. Acta, 1996, 35, 202-209.

[14] Koran, F.; Dealy , J. M. A high pressure sliding plate rheometer for polymer melts, J. Rheol., 1999, 43, 1279-1290.

[15] Barati, R.; Liang, J. A review of fracturing fluid systems used for hydraulic fracturing of oil and gas wells. J. Appl. Polym. Sci. 2014, 131(16), 40735.

[16] Peng, H. ; Zhou, S.; Jiang, J.; Guo, T.; Zheng, X.; Yu, X. Pressure-Induced Crystal Memory Effect of Spider Silk Proteins, J. Phys. Chem. B. 2009, 113, 4636-4641.

[17] Rastogi, N. K.; Raghavarao, K. S. M. S.; Balasubramaniam, V. M.; Niranjan, K.; Knorr, D. Opportunities and challenges in high pressure processing of foods. Crit. Rev. Food Sci. Nutr. 2007, 47(1), 69-112.

[18] Appel, E. A.; Biedermann, F.; Hoogland, D.; del Barrio, J.; Driscoll, M. D.; Hay, S.; Wales, D. J.; Scherman, O. A. Decoupled Associative and Dissociative Processes in Strong yet Highly Dynamic Host-Guest Complexes. J. Am. Chem. Soc. 2017, 139, 12985-12993.

[19] Ducouret, G. ; Chassenieux, C. ; Martins, S. ; Lequeux, F. ; Bouteiller, L. Rheological characterisation of bis-urea based viscoelastic solutions in an apolar solvent. J. Colloid Interf. Sci. 2007, 310, 624-629.

[20] Lortie, F. ; Boileau, S. ; Bouteiller, L. ;Chassenieux, C. ; Demé, B. ; Ducouret, G. ; Jalabert, M. ; Lauprêtre, F.; Terech, P. Structural and rheological study of a bis-urea based reversible polymer in an apolar solvent. Langmuir 2002, 18, 7218-7222.

[21] Simic, V.; Bouteiller, L.; Jalabert, M. Highly cooperative formation of bis-urea based supramolecular polymers. J. Am. Chem. Soc. 2003, 125, 13148-13154.

[22] Bellot, M.;Bouteiller, L. Thermodynamic description of bis-urea self-assembly: competition between two supramolecular polymers. Langmuir 2008, 24, 14176-14182. [23] Shikata, T.;Nishida, T.; Isare, B.; Linares, M.; Lazzaroni, R.; Bouteiller, L. Structure and dynamics of a bisurea-based supramolecular polymer in n-dodecane. J. Phys. Chem. B 2008, 112, 8459-8465.

[24] Sabadini, E.; Francisco, K. R.; Bouteiller, L. Bis-urea-based supramolecular 
polymer: The first self-assembled drag reducer for hydrocarbon solvents. Langmuir 2009, 26, 1482-1486.

[25] Francisco, K. R.; Dreiss, C. A.; Bouteiller, L.; Sabadini, E. Tuning the Viscoelastic Properties of Bis (urea)-Based Supramolecular Polymer Solutions by Adding Cosolutes. Langmuir 2012, 28, 14531-14539.

[26] Van der Gucht, J.; Besseling, N.; Knoben, W.; Bouteiller, L.; Stuart, M. C. Brownian particles in supramolecular polymer solutions. Phys. Rev. E 2003, 67, 051106.

[27] Louhichi, A.; Jacob, A. R.; Bouteiller, L.; Vlassopoulos, D. Humidity affects the viscoelastic properties of supramolecular living polymers. J. Rheol., 2017, 61, 1173-1182. [28] Jacob, A. R.; Deshpande, A. P.; Bouteiller, L. Large amplitude oscillatory shear of supramolecular materials. J. Non-Newtonian Fluid Mech. 2014, 206, 40-56.

[29] Pinault, T.; Isare, B. ; Bouteiller, L. Solvents with similar bulk properties induce distinct supramolecular architectures. Chem. Phys. Chem. 2006, 7, 816-819. [30] Hendricks, J. ; Louhichi, A. ; Merti, V. ; Fournier, R. ; Reddy, N. ; Bouteiller, L. ; Cloitre, M. ; Clasen, C. ; Vlassopoulos, D.; Briels, W. J. Non-monotonic stress relaxation after cessation of steady shear flow in supramolecular assemblies. Phys. Rev. Lett. 2019, 123, 218003.

[31] Hansch, M.; Ranfta, M. Dhuwe, A.; Enick, R. Thickening compressed liquid and supercritical propane with bisurea DMHUT N,N'-(4-methyl-1,3-phenylene)bis[N-(1,5dimethylhexyl)urea] for enhanced oil recovery or waterless hydraulic fracturing. J. Supercr. Fluids 2019, 145, 85-92.

[32] Reichardt, C.; Welton, T. Solvents and Solvent Effects in Organic Chemistry, Wiley-VCH Publishers, $4^{\text {th }}$ ed., 2011.

[33] Meier, G.; Kriegs, H. A high pressure cell for dynamic light scattering up to 2kbarswith conservation of plane of polarization. Rev. Sci. Instr. 2008, 79, 013102.

[34] Meier, G.; Vavrin, R.; Kohlbrecher, J. ; Buitenhuis, J.; Lettinga, M. P.; Ratajczyk, M. SANS and dynamic light scattering to investigate the viscosity of toluene under high pressure up to 1800 bar. Mater. Sci. Technol. 2008, 19, 034017.

[35] Ferrell, R. T. ; Himmelblau, D. M. Diffusion Coefficients of Nitrogen and Oxygen in Water, J. Chem. Eng. Data 1967, 12, 111-115. 
[36] Dennis, K.; Gao, Y.; Phathak, A.; Sullivan, P. F. ; Furst, E. M. Design, operation, and validation of a microrheology instrument for high-pressure linear viscoelasticity measurements. J. Rheol. 2020, 64, 205-212.

[37] Furst, E. M. ; Squires, T. M. Microrheology, Oxford University Press, 2017.

[38] Royall, C. P. ;Poon, W. C. K.; Weeks, E. R. In search of hard spheres. Soft Matter, 2013, 9, 17-27.

[39] Schärtl, W. Light Scattering from Polymer Solutions and Nanoparticle Dispersions (Springer Science \& Business Media, 2007).[40] BIOVIA, Dassault Systèmes, Biovia Materials Studio, 6.0, San Diego: Dassault Systèmes, 2011.

[41] Brocorens, P.; Linares, M.; Guyard-Duhayon, C.; Guillot, R. ; Andrioletti, E.; Suhr, D.; Isare, B.; Lazzaroni, R.; Bouteiller, L. Conformational Plasticity of Hydrogen Bonded Bis-urea Supramolecular Polymers. J. Phys. Chem. B. 2013, 117(17), 5379-5386.

[42] Mayo, S. L.; Olafson, B. D.; Goddard III., W. A. DREIDING: A Generic Force Field for Molecular Simulations. J. Phys. Chem. 1990, 94, 8897-8909.

[43] Alvarenga, B. G.; Raynal, M.; Bouteiller, L.; Sabadini, E. Unexpected Solvent Influence on the Rheology of Supramolecular Polymers. Macromolecules 2017, 50, 6631-6636.

[44] Bouteiller, L.; Colombani, O.; Lortie, F.;Terech, P. Thickness transition of a rigid supramolecular polymer. J. Am. Chem. Soc. 2005, 127, 8893-8898.

[45] Jian, T. ; Vlassopoulos, D. ; Fytas, G.; Pakula, T.; Brown, W. Coupling of Concentration Fluctuations to Viscoelasticity in Highly Concentrated Polymer Solutions. Colloid Polym Sci. 1996, 274, 1033-1043.

[6] Nicolai, T.; Brown, W.; Johnsen, R. M.; Stepanek, P. Dynamic Behavior of Theta Solutions of Polystyrene Investigated by Dynamic Light-Scattering. Macromolecules 1990, 23, 1165 1174.

[47] Wang, J. ;Wu, C. Reexamination of the Origin of Slow Relaxation in Semidilute Polymer Solutions-Reptation Related or Not?. Macromolecules 1990, 49, 3184-3191.

[48] Willenbacher, N.; Oelschlaeger, C. ; Schopferer, M. ; Fischer, P.; Cardinaux, F.;

Scheffold, F. Broad Bandwidth Optical and Mechanical Rheometry ofWormlike Micelle

Solutions. Phys. Rev. Lett. 2007, 99, 068302.

[49] Willenbacher, N.; Oelschlaeger, C. Dynamics and structure of complex fluids from high 
frequency mechanical and optical rheometry. Curr. Opinion Colloid Interface Sci. 2007, 12, 43-49.

[50] Bouteiller, L.; van der Schoot, P. Probing Weak Intermolecular Interactions in SelfAssembled Nanotubes. J. Am. Chem. Soc. 2012, 134, 1363-1366. 